\title{
Would General Practitioners Provide Correct Information To Mothers And Fathers Of Infants? An Assessment Of The Knowledge Base Related To Breastfeeding And Kangaroo Mother Care
}

Somashekhar Nimbalkar, Nitesh Sadhwani

Department of Pediatrics, Pramukhswami Medical College, Karamsad, Gujarat, India. Pin -388325

Central Research Services, Charutar Arogya Mandal, Karamsad, Gujarat, India. Pin - 388325

Email: somu somu@yahoo.com. Phone: 9825087842

\section{Background:}

Breastfeeding is the ideal method suited for the physiological and psychological needs of an infant.

More than $15 \%$ of 24 lakh child deaths could be averted in India by optimal breastfeeding practices.

Kangaroo mother care (KMC) can prevent most of the deaths in this population and the infants can have superior developmental outcomes lasting into adult life.

General practitioners are first line of contact in majority of population.

Need of proper counseling regarding breastfeeding and kangaroo mother care is of utmost important for overall growth and development of baby.

Aim:

To assess the completeness of basic knowledge of breastfeeding and kangaroo mother care in general practitioners in Anand district of Gujarat.

\section{Methods:}

We approached $\mathbf{1 8 0}$ doctors in and around the Anand who are either allopathic, homeopathic or ayurvedic practitioners of healthcare.

They filled out a survey questionnaire which asked them to provide answers related to breastfeeding and kangaroo mother care.

Data was analyzed by descriptive statistics.

\section{Results:}

158 doctors responded (41 MBBS, 85 BHMS and 32 BAMS). $69 \%$ believed breastfeeding should be started as soon as possible with $91 \%$ believe colostrums is good for baby.

$>72 \%$ believed breastfeeding is not enough and animal milk water, honey and other fluids to be given.

Only $56 \%$ believe breastfeeding is exclusively given for 6 months.

$70 \%$ believe formula feed is more convenient than breastfeeding.

$>9 \%$ knew all signs of good attachment and $12 \%$ knew all signs of good positioning.

$78 \%$ believe breastfeeding should be avoided when mother is sick and $68 \%$ believe it is to be omitted when baby is sick.

Only $21 \%$ knew about KMC but most of them did not know about benefits of kangaroo mother care and proper technique and hence counselling was not done regarding kangaroo mother care.

$\rightarrow$ Major source of knowledge being books \& journals(22\%) and other doctors $(21 \%)$

$>31 \%$ knew it's role in hypothermia and $77 \%$ believed it decreased hospital stay, $68 \%$ knew any member can give kmc, $77 \%$ believe it helps in relieving pain, $71 \%$ believe it increases breastmilk secretion.

\section{Conclusion:}

Simple survey shows that there is a wide gap in the knowledge fund of general practitioners.

Interventions need to be designed to get them up to speed so that the infants get the best care provided care by them. Updating knowledge of general practitioners may be helpful in growth and development of babies. 